\title{
Smoking supernovae
}

\author{
H.L. Gomez', S.A. Eales' and L. Dunne ${ }^{2}$ \\ ${ }^{1}$ School of Physics and Astronomy, Cardiff University, Queens Buildings, The Parade, Cardiff, Wales CF24 3AA, UK \\ e-mail:haley.gomez@astro.cf.ac.uk \\ ${ }^{2}$ School of Physics and Astronomy, University of Nottingham, University Park, Nottingham NG7 2RD, UK
}

\begin{abstract}
The question 'Are supernovae important sources of dust?' is a contentious one. Observations with the Infrared Astronomical Satellite (IRAS) and the Infrared Space Observatory (ISO) only detected very small amounts of hot dust in supernova remnants. Here, we review observations of two young Galactic remnants with the Submillimetre Common User Bolometer Array (SCUBA), which imply that large quantities of dust are produced by supernovae. The association of dust with the Cassiopeia A remnant is in question owing to the contamination of foreground material. In this paper, we compare the emission from cold dust with $\mathrm{CO}$ emission towards Kepler's supernova remnant. We detect very little $\mathrm{CO}$ at the location of the submillimetre peaks. A comparison of masses from the $\mathrm{CO}$ and the dust clouds are made, and we estimate the $3 \sigma$ upper limit on the gas-to-dust ratios to be in the range 20-60. These results suggest that we cannot yet rule out freshly-formed or swept-up circumstellar dust in Kepler's supernova remnant.

Received 1 December 2006, accepted 28 December 2006
\end{abstract}

Key words: astronomy, Cassiopeia A, interstellar dust, Kepler, supernovae.

\section{Introduction}

Interstellar dust plays an important role in astronomy, yet we know relatively little about the origin and evolution of the dust cycle in galaxies. Although dust grains only constitute around $1 \%$ of the mass of the interstellar medium (ISM), they affect our view of the Universe by blocking out optical light and changing the visible appearance of astrophysical objects. Dust grains scatter, absorb and re-emit light to longer wavelengths so effectively that observing techniques at wavelengths other than optical are needed to obtain a complete picture of the Universe. Recently, astronomers have realized that dust is far more than the 'smoke' between the stars, particularly since the advent of infrared and submillimetre (sub-mm) telescopes which directly detect the recycled emission from dust grains. Surveys have shown that dust plays an important part in the cooling processes of the gas and its interaction with the gas dynamically, as well as providing greater understanding of stellar chemistry; indeed, it is believed to be the main catalyst for the formation of molecular hydrogen in space (Hirashita \& Ferrara 2002), an effective coolant in star forming regions, an important tracer of metals in the Universe (Dunne et al. 2003a) and a possible tracer for high-density gas. Perhaps the most convincing argument for the importance of understanding the origin and evolution of dust is seen in the recent studies of the infrared and sub-mm background. These observations clearly show that the amount of energy in the infrared/sub-mm background is almost as much as that in the optical background (Fig. 1). This has one serious implication-almost half of all the optical light emitted since the Big Bang has been absorbed and re-radiated by dust.

Although we recognize the importance of interstellar dust, even the source of dust in the Galaxy is unknown. There are many observations which provide evidence for dust grain formation in stellar outflows: infrared emission around red giants, planetary nebulae, Wolf-Rayet stars and carbon stars. Indeed, stellar winds (SWs) are thought to be the most important contributors to stardust into the ISM (Jones et al. 1996; Draine 2003; Whittet 2003). The question is, how much? Dust production in stars is hard to quantify observationally, it depends on the mass of heavy elements in the stellar atmospheres and the mass loss rates during the final stages of the star's evolution. The required cycle to produce dust in stars begins with the enrichment of the ISM from the first population of rapidly evolving supernovae $(\mathrm{SNe})$, the incorporation of these elements into star formation and the evolution of the stars to the right atmospheric conditions before significant dust production can occur. The timescale for dust injection from stars is of the order of a few billion years. Observations of mass loss rates from intermediate mass stars suggest that they contribute $86-97 \%$ of the total dust mass injected from astrophysical sources (Whittet 2003). However, there is a major problem with this statement: there is not enough dust in SWs to explain the mass of dust we see in our own Galaxy (known as the dust budget crisis). This problem is further compounded by the recent discovery of a population of extremely dusty objects at high redshifts seen in blank field sub-mm surveys and observations of distant quasars (Bertoldi et al. 2003; Eales et al. 2003) which imply 


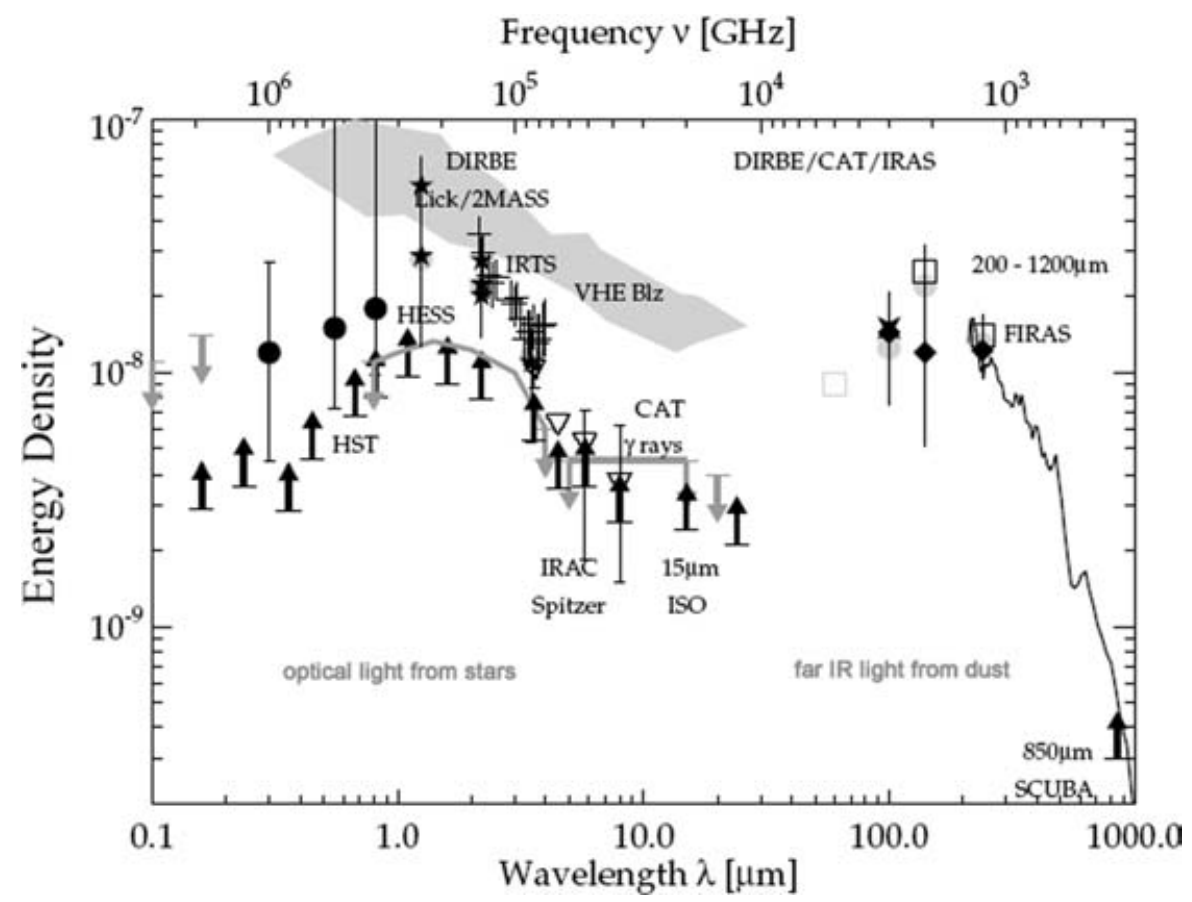

Fig. 1. The integrated background energy in the optical-infrared regime (adapted from Dole et al. 2006). Black arrows represent lower limits. Starlight and dust reprocessed starlight contribute almost equal amounts to the background energy.

that dusty galaxies are present in the Universe at $z>4$ (Smail et al. 1997; Isaak et al. 2002). The Universe was less than $1 / 10$ th of its present day age at this time and it is difficult for the dust to have originated from the SWs of intermediate mass stars in such short timescales. An alternative source of dust could be $\mathrm{SNe}$, as they provide large abundances of heavy elements and can create the required density/temperature/ pressure conditions for dust to condense (Clayton et al. 2001; Todini \& Ferrara 2001; Nozawa et al. 2003). Type-II SNe are the explosions of massive stars, which evolve rapidly and reach the supernova phase after only 10-100 Myr. Thus, a Type-II SNe could potentially provide a rapid source of dust. If there is little or no dust formation from rapidly evolving $\mathrm{SNe}$ then it is difficult to understand where the high-redshift dust originated. This problem is highlighted in Fig. 2 where the evolution of dust mass for a galaxy $\left(10^{10} M_{\text {solar }}\right)$ is shown using a theoretical chemical evolution model to show how the dust builds up with time (Morgan \& Edmunds 2003). The two solid lines represent dust mass from $\mathrm{SNe}$ and $\mathrm{SWs}$. If $\mathrm{SNe}$ are not important contributors to the interstellar dust budget, it will take this galaxy at least five billion years $(z<2)$ to build up a dust mass of greater than $10^{7} M_{\text {solar }}$ from SWs only. The mass of dust seen in high- $z$ galaxies and quasar systems at $z>4$ is around $10^{8} M_{\text {solar }}$. These problems suggest that dust formation in supernovae (or, more importantly, a rapid source of dust) is required to explain both the presence of high-redshift dust and the dust mass in our own Galaxy.

Theorists have long championed dust formation in supernovae (e.g. Clayton et al. 2001; Todini \& Ferrara 2001; Nozawa et al. 2003) yet observations with infrared cameras

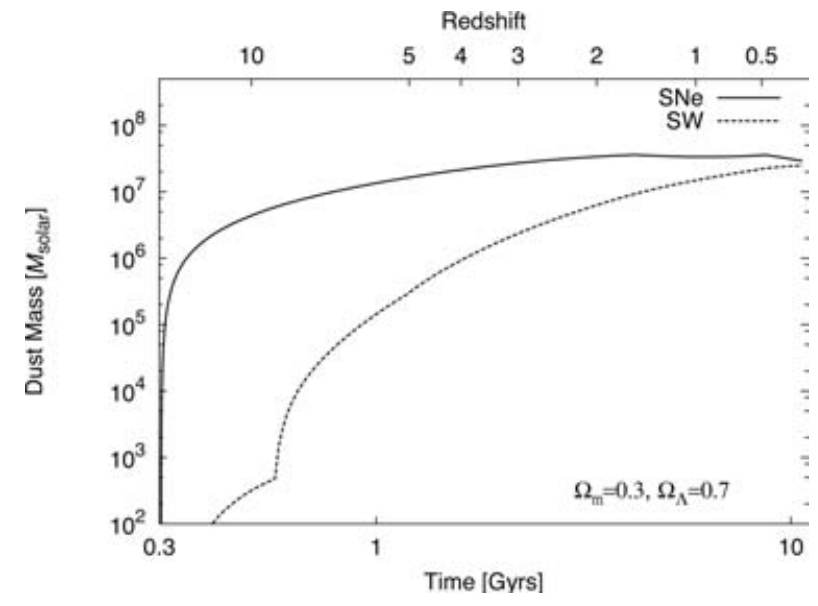

Fig. 2. The dust evolution of a galaxy $\left(10^{10} M_{\text {solar }}\right)$ with time with star formation rate $1 M_{\text {solar }} \mathrm{yr}^{-1}$ (Morgan \& Edmunds 2003). Redshifts are calculated using the concordance cosmological model, $\Omega_{\mathrm{m}}=0.3$ and $\Omega_{\Lambda}=0.7$. In this case the contributions from dust formation in $\mathrm{SNe}$ using theoretical estimates (e.g. Todini \& Ferrara 2001) and observations of SWs (Whittet 2003) are shown separately as solid and dotted lines, respectively.

such as the Infrared Astronomical Satellite (IRAS) showed very little amounts of dust observationally. In one galactic supernova remnant (SNR), Cassiopeia A (Cas A), astronomers observed a tiny $10^{-7} M_{\text {solar }}$ of dust with IRAS. Later observations with the Infrared Space Observatory (ISO) hinted at the presence of much more dust, finding $0.15 M_{\text {solar }}$ (Tuffs et al. 1999), but the low resolution of ISO and 


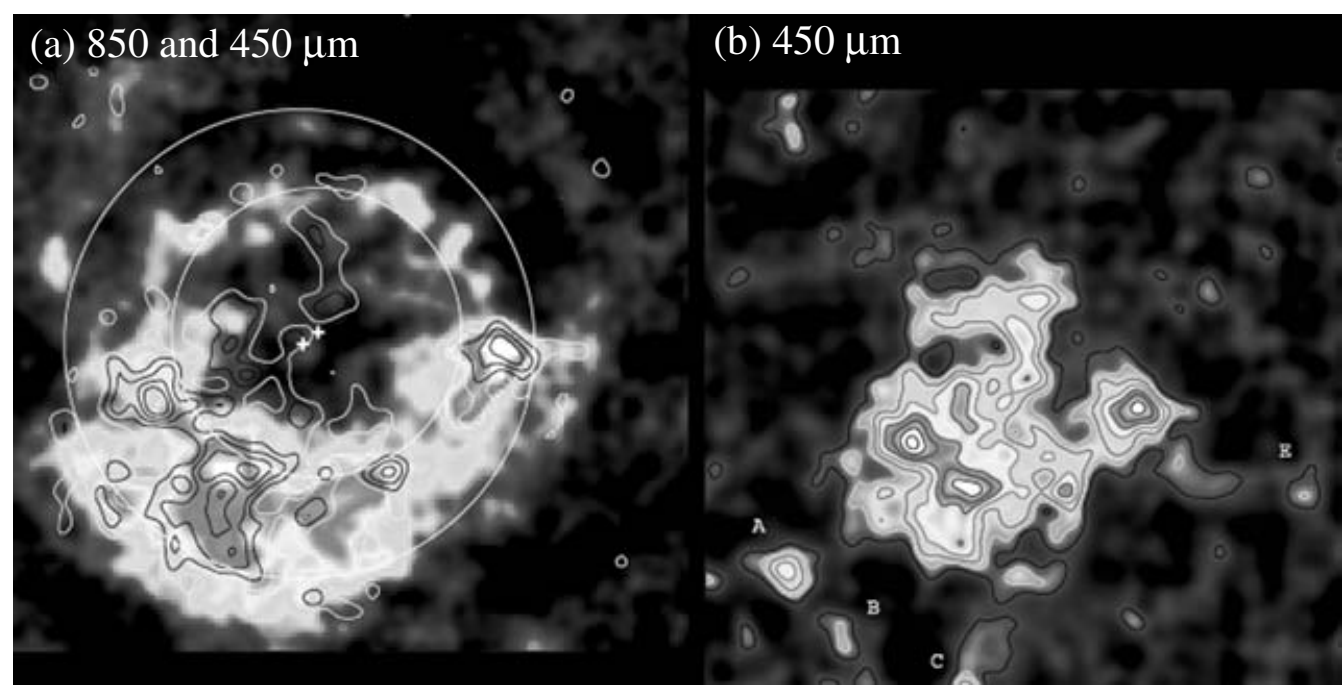

Fig. 3. SCUBA images of the Cas A supernova remnant at (a) $850 \mu \mathrm{m}$ and (b) $450 \mu \mathrm{m}$ (from Dunne et al. 2003b). In (a) we show the synchrotron-subtracted $850 \mu \mathrm{m}$ image with $450 \mu \mathrm{m}$ contours overlaid $(3 \sigma+1 \sigma)$. The forward and reverse shock fronts seen in the X-ray from the supernova blast wave are overlaid (Gotthelf et al. 2001).

instrumental difficulties at the longer wavelengths meant that this result was largely ignored. The total dust yield from $\mathrm{SNe}$ estimated using the observations is no more than $10^{-5}$ $M_{\text {solar }}$ of dust per year, whereas the models imply that they could inject anywhere between $0.4 \times 10^{-3}$ and $40 \times 10^{-3}$ $M_{\text {solar }}$ of dust per year. The huge discrepancy between the theoretical models and observations of $\mathrm{SNe}$ could be explained if there existed a population of cold dust in the $\mathrm{SNe}$ ejecta not visible with infrared telescopes, which are sensitive to emission from hot dust. Such a population of cold dust grains would emit at longer, submillimetre (sub-mm) wavelengths. The Submillimetre Common User Bolometer Array (SCUBA) camera on the James Clerk Maxwell Telescope (JCMT) in Hawaii was used to observe two young supernova remnants, Cas A and Kepler, at 850 and $450 \mu \mathrm{m}$. (To determine if the dust is freshly formed by the $\mathrm{SNe}$ explosion or blast wave, we require observations of 'young' remnants that are still dominated by the ejecta dynamics, i.e. have not swept up much gas. This limits us to very small numbers of possible sources, further compounded by the poor sensitivity of SCUBA and the fact that most of these objects lie towards the centre of the Galaxy and are confused by foreground material.) These observations discovered large amounts of sub$\mathrm{mm}$ emission, suggesting three orders of magnitude more dust existed in the remnants than seen with IRAS and ISO. Recent observations have suggested that there may be alternative explanations for the large dust masses detected with SCUBA.

In this paper, we review the original sub-mm observations and data reduction of these two remnants, including a detailed description of how the dust mass was estimated. We compare the sub-mm observations with our new carbon monoxide (CO) images towards the remnants to determine if there is contamination from foreground molecular clouds. We also review whether or not the SCUBA dust could be from an 'exotic' form of dust grains, in the shape of iron needles. Finally we discuss the consequences of the $\mathrm{CO}$ and sub-mm observations.

\section{Submillimetre observations of supernovae - Cassiopeia A}

Cas A is the brightest radio source in the sky and is believed to be the remnant of a massive star which exploded around 300 years ago. It lies at a distance of approximately $3.5 \mathrm{kpc}$ with diameter of approximately 8 arcmin. The SCUBA observations were made in 1995 in the scan-mapping mode and were available in the JCMT archive. After data reduction, low-level regions of diffuse emission remained on the image. Scan mapping typically leaves such artefacts and they vary depending on the methods chosen for removing the baselines. Therefore, a surface was fitted to the image, which left the background flat. This was checked by taking extra photometry data (not prone to the same systematics as scan mapping) in December 2002 at 850 and $450 \mu \mathrm{m}$, which provided an independent check of the absolute flux levels at several positions on the remnant.

The SCUBA images of Cas A are shown in Fig. 3 at (a) $850 \mu \mathrm{m}$ with $450 \mu \mathrm{m}$ contours overlaid $(3 \sigma+1 \sigma)$ and (b) $450 \mu \mathrm{m}$ (Dunne et al. 2003b). Around two-thirds of the emission at $850 \mu \mathrm{m}$ is contaminated with synchrotron emission described by a power-law slope $v^{-\alpha}$. Once this component is subtracted, we can see the emission from cold dust only; Fig. 3(a) shows the synchrotron-subtracted $850 \mu \mathrm{m}$ image. The forward and reverse shock fronts seen in the X-ray from the supernova blast wave are also overlaid (Gotthelf et al. 2001). Once the synchrotron emission is subtracted the 850 and $450 \mu \mathrm{m}$ emissions follow a similar distribution with the cold dust now located mainly in the south and eastern parts of the remnant. The sub-mm peaks appear to fall 


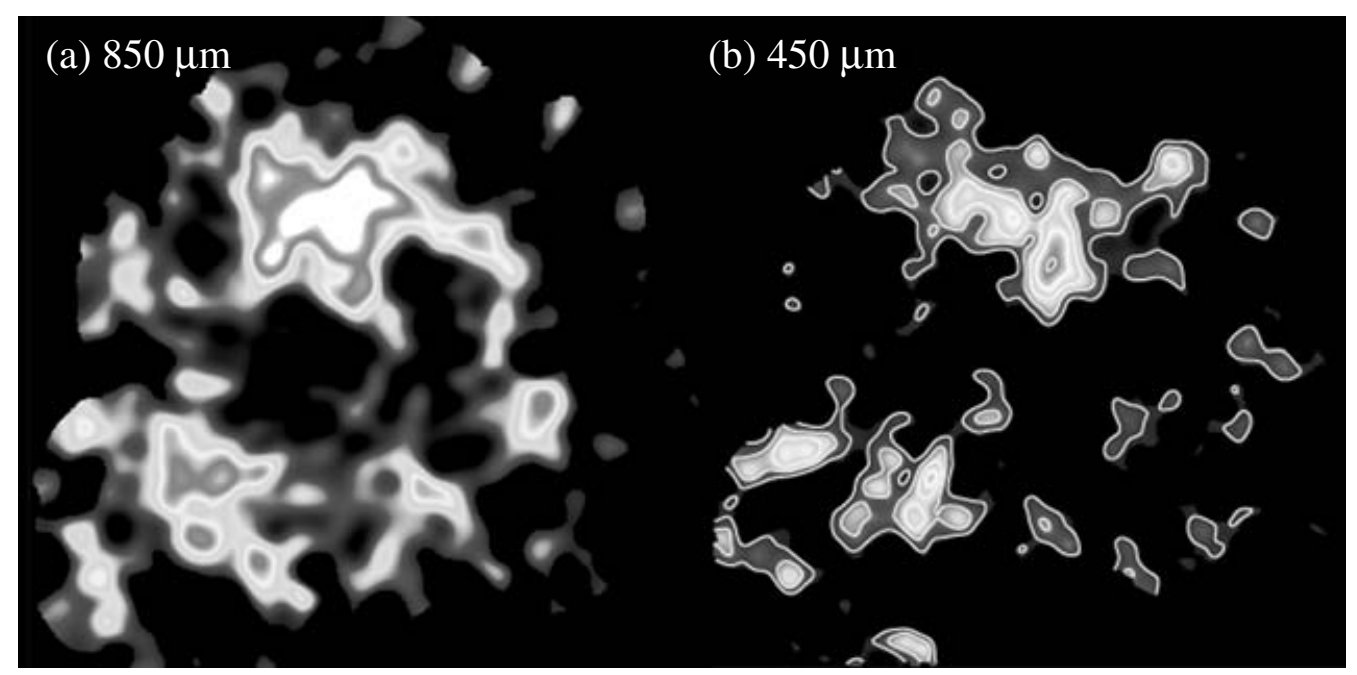

Fig. 4. SCUBA images of Kepler's supernova remnant at (a) $850 \mu \mathrm{m}$ and (b) $450 \mu \mathrm{m}$ (Morgan et al. 2003). The original reduced data maps have been divided by a simulated noise map, these images therefore represent a signal-to-noise map of Kepler.

between the forward and reverse shocks seen in Fig. 3(a). The final integrated sub-mm fluxes for Cas A's SNR, minus the synchrotron component (i.e. from cold dust), are $S_{850 \mu \mathrm{m}} \sim 15.8 \pm 5.6 \mathrm{Jy}$ and $S_{450 \mu \mathrm{m}} \sim 47.5 \pm 16.1 \mathrm{Jy}$.

\section{Kepler}

The explosion in 1604 left behind a shell-like remnant $\sim 3$ arcmin in diameter which lies at a distance of approximately $5 \mathrm{kpc}$. The progenitor and supernova type is controversial (Schaefer 1996; Blair 2004). There is dynamical evidence to suggest that the explosion was a Type II (massive star explosion) along with an overabundance of nitrogen thought to be made from the CNO cycle of massive stars (see Borkowski et al. 1992), but model-fitting to X-ray spectra suggests the ejecta has a chemical composition similar to that expected from a Type Ia explosion (the nuclear explosion from a white dwarf binary system, see Kinugasa \& Tsunemi (1999)).

Six 'jiggle-map' observations were centred upon the SNR since the remnant is larger than the SCUBA's fieldof-view, with chop throw 180 arcsec. Figure 4 shows the SCUBA signal-to-noise images of Kepler at (a) $850 \mu \mathrm{m}$ and (b) $450 \mu \mathrm{m}$ (Morgan et al. 2003); the shell-like structure is clearly visible at $850 \mu \mathrm{m}$. The synchrotron component is far less in Kepler as it is not as radio bright as Cas A. The final integrated sub-mm fluxes for Kepler's SNR, minus the synchrotron component, are $S_{850 \mu \mathrm{m}} \sim 1.0 \pm 0.16 \mathrm{Jy}$ and $S_{450 \mu \mathrm{m}} \sim 3.0 \pm 0.7 \mathrm{Jy}$.

\section{Estimating the dust mass}

The dust mass can be measured directly from the flux at submm wavelengths using (Hildebrand 1983)

$M_{\mathrm{d}}=\frac{S_{v} D^{2}}{\kappa_{v} B\left(v, T_{\mathrm{d}}\right)}$ where $S_{v}$ is the flux density measured at frequency $v, D$ is the distance and $\kappa_{v}$ is the dust mass absorption coefficient. $B(v, T)$ is the Planck function and $T_{\mathrm{d}}$ is the dust temperature. We fitted a two-temperature grey body to the infrared-sub-mm spectral energy distribution (SED) of the two remnants, allowing the dust emissivity parameter, $\beta$, and the warm and cold temperatures to vary. The best-fit SEDs for Kepler and Cas A are shown in Figs 5 and 6, respectively, with the best-fit parameters for each SNR listed in the captions. We used a bootstrap technique to derive errors on these values, creating 3000 sets of artificial fluxes from the original fluxes and their associated error bars. Our two-temperature model was then applied to each artificial set and errors derived from the distribution of $T_{\text {warm }}, T_{\text {cold }}$ and $\beta$ produced by these fits (inset).

The largest uncertainty in the dust mass comes from the uncertainty in $\kappa_{v}$ We have followed Dunne et al. (2003a) in trying three different values of $\kappa_{v}$ from the literature: (1) $\kappa_{850 \mu \mathrm{m}} \sim 0.85 \mathrm{~m}^{2} \mathrm{~kg}^{-1}$, the average value from the range observed in laboratory studies of clumpy aggregates; (2) $\kappa_{850 ~ u \mathrm{~m}} \sim 0.48 \mathrm{~m}^{2} \mathrm{~kg}^{-1}$, the average observed in circumstellar environments; and (3) $\kappa_{850} \mathrm{um} \sim 0.01 \mathrm{~m}^{2} \mathrm{~kg}^{-1}$, the average observed for the diffuse ISM where dust is likely to have encountered extensive processing. In Cas A, the higher $\kappa_{850} \mu \mathrm{m}$ values were required to give a reasonable dust mass of $2.6 \pm 0.7 M_{\text {solar }}$. If we used the $\kappa$ values relevant for 'normal' interstellar dust, the dust mass is uncomfortably large, being greater than $15.0 \pm 4 M_{\text {solar }}$ Using the laboratory $\kappa$ values for Kepler gives a lower limit of $0.3 \pm 0.1 M_{\text {solar }}$, whereas using the 'normal' dust $\kappa$ values gives $2.7 \pm 0.6$ $M_{\text {solar }}$

\section{Alternative explanations}

Given the importance of determining the correct mass of dust produced by $\mathrm{SNe}$ or their massive star progenitors, two competing theories have been put forward claiming that the 


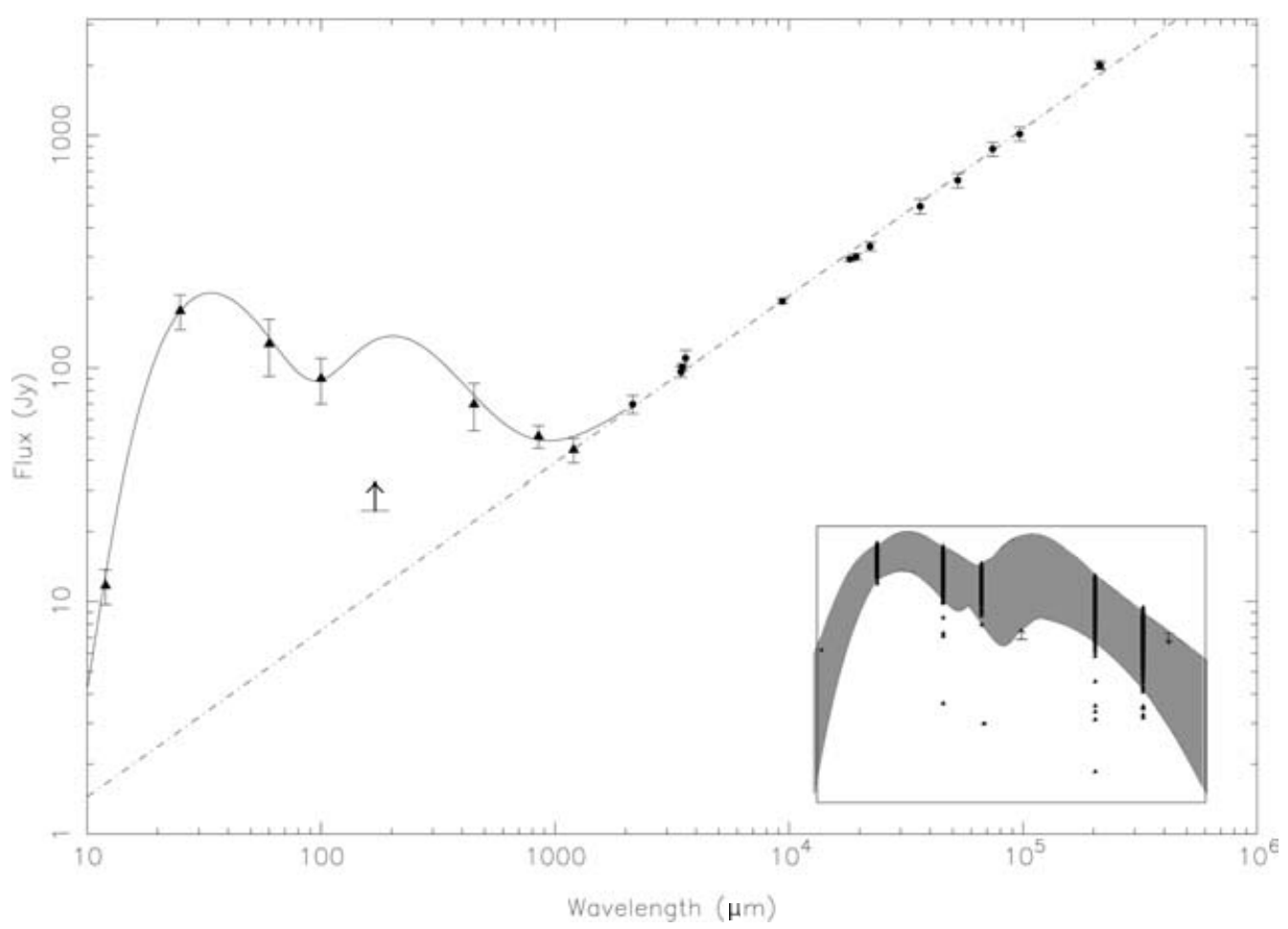

Fig. 5. The spectral energy distribution of Cas A. The solid lines represent the two-temperature-best-fit $\chi^{2}$ test, with dot-dashed lines representing the hot and cold component of dust grains in each SED. The $170 \mu \mathrm{m}$ lower limit from ISO is shown (Tuffs et al. 1999). The inset shows the 3000 fits from the bootstrap technique from the original data points (those with $\chi^{2}<3.0$ are shown). Although there is no data point to confirm the existence of the second peak around 200-400 $\mu \mathrm{m}$, it appears to remain even when fitting extremes to the SED. Best fit parameters are $T_{\text {hot }} \sim 112_{-21}^{+11} \mathrm{~K}, T_{\text {cold }} \sim 17 \pm 3.6 \mathrm{~K}, \beta \sim 0.9_{-0.6}^{+0.8}$ with radio power law of $v^{-0.61}$.

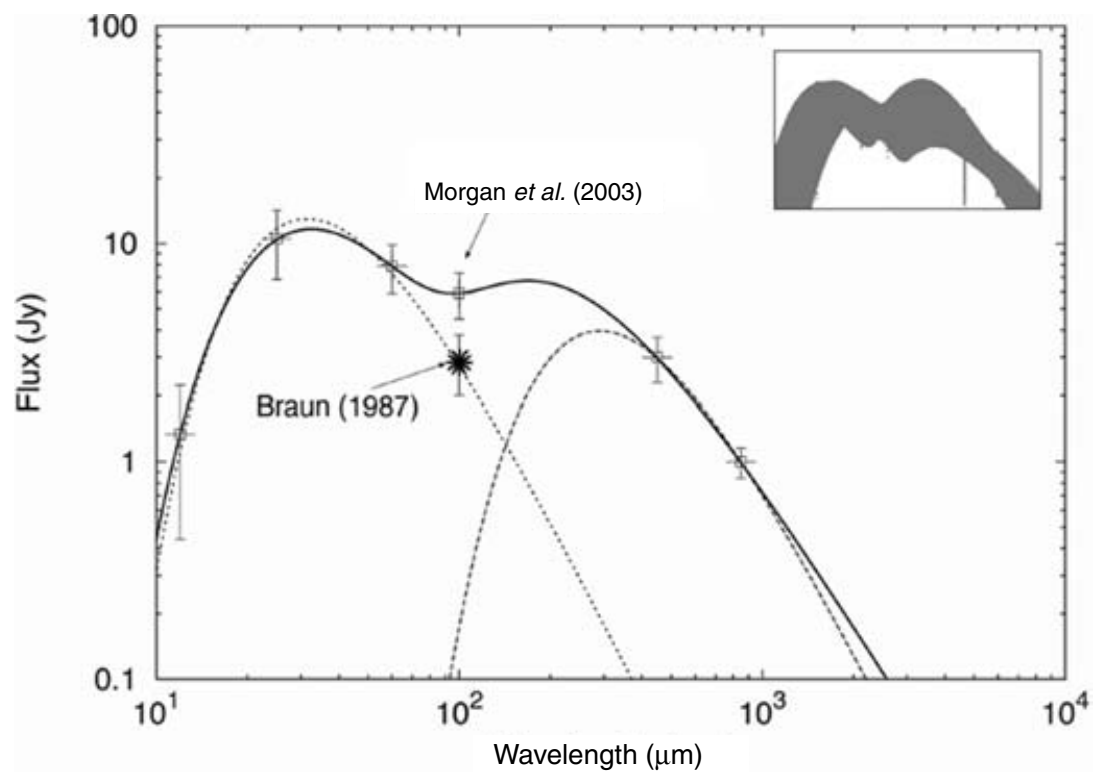

Fig. 6. The spectral energy distribution of Kepler. Two 'best' fits to the infrared-sub-mm data for Kepler are shown owing to the uncertainty in IRAS fluxes for Kepler at $100 \mu \mathrm{m}$ ranging from $2.9 \mathrm{Jy}$ (value from pointed observations of the remnant (Braun et al. 1987)) to 5.9 Jy (the average of all values for Kepler published with IRAS). Neither fit rules out a cold dust component. The inset shows the results from the bootstrap technique. The best-fit parameters are $T_{\text {hot }} \sim 102 \pm 12 \mathrm{~K}, T_{\text {cold }} \sim 17_{-3}^{+2} \mathrm{~K}, \beta \sim 1.2 \pm 0.4$ with radio power law of $v^{-0.71}$.

dust mass in these remnants is in fact much lower: (i) 'exotic' needle-like metallic grains are responsible for the dust emission; and (ii) the emission in Cas A is contaminated by foreground material and is not associated with the remnant. In this section, we discuss the evidence for and against both theories and their possible implications. 
(a)

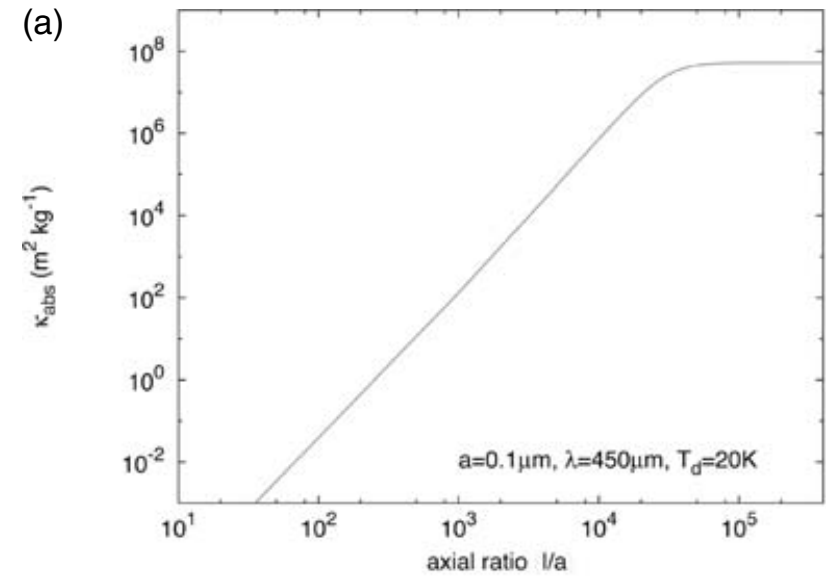

(b)

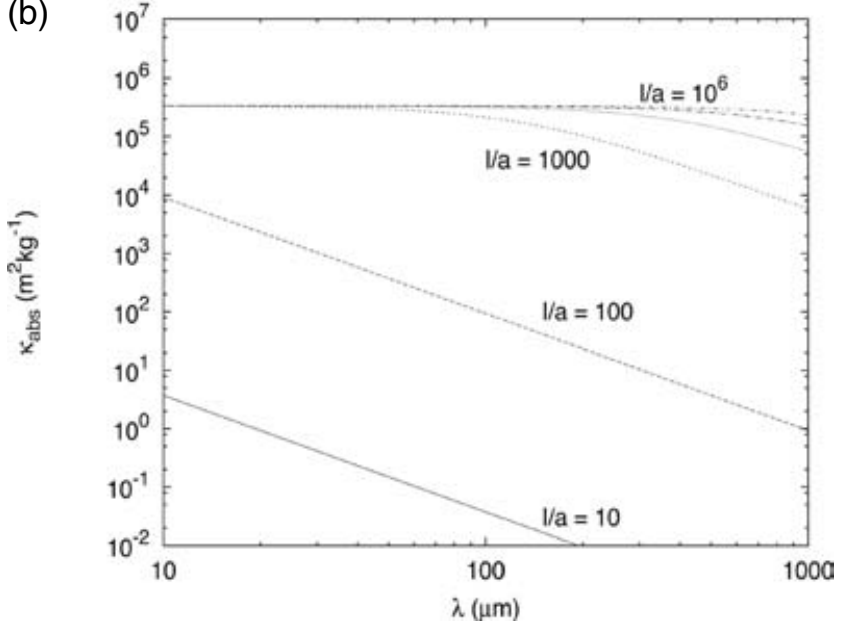

Fig. 7. (a) The variation of the absorption coefficient of an iron needle with axial ratios $(l / a)$ for typical interstellar grain size, $a=0.1 \mu \mathrm{m}$, at $450 \mu \mathrm{m}$ (following Li 2003). (b) The variation of the absorption coefficient of iron needles with wavelength for different axial ratios $(l / a)$. The needles are modelled as antenna with a resistivity of $10^{-5} \Omega \mathrm{cm}$ (Gomez et al. 2005).

\section{'Exotic' dust grains}

Conducting iron needles were proposed as an alternative explanation for the emission in the SCUBA image of Cas A (Dwek 2004). Such needles, if they exist, would be efficient emitters at sub-mm wavelengths and would be collisionally heated by the hot X-ray gases in the supernova blast wave to temperatures of around $10 \mathrm{~K}$. The high emissivity of the needles gives rise to large absorption coefficients, which serve to decrease the dust mass determined from the emission by several orders of magnitude (e.g. Edmunds \& Wickramasinghe 1975). The dust mass absorption coefficient for iron needles is given by

$\kappa=\frac{4 \pi}{3 c \rho_{\mathrm{d}} \rho_{r}}$

where $\rho_{\mathrm{d}}$ is the density of iron and $\rho_{r}$ is the conductivity. The variation of the absorption coefficient of an iron needle with axial ratio (length/radius, $l / a$ ) for a radius of $0.1 \mu \mathrm{m}$ at $450 \mu \mathrm{m}$ is show in Fig. 7(a). The variation of the absorption coefficient of iron needles with wavelength for different axial ratios is plotted in Fig. 7(b). The needles are modelled as antenna with a resistivity of $10^{-5} \Omega \mathrm{cm}$. The absorption coefficient at $850 \mu \mathrm{m}$ for iron needles with $l / a \sim 10000$ is $\sim 10^{5} \mathrm{~m}^{2} \mathrm{~kg}^{-1}$. For comparison, the absorption coefficient at $850 \mu \mathrm{m}$ for 'normal' interstellar dust is $\sim 0.07 \mathrm{~m}^{2} \mathrm{~kg}^{-1}$. Given that $M_{\mathrm{d}} \propto 1 / \kappa$, Dwek (2004) estimated that the mass of iron needles required to explain the sub-mm emission from Cas A would only be $\sim 10^{-5} M_{\text {solar }}$. Using Dwek's formulization of the heating and cooling of the needles in the supernova blast wave, we investigated whether or not the emission from Kepler's remnant could be explained by these 'exotic' dust grains (Gomez et al. 2005). We found that the mass of iron needles required to explain the sub-mm emission from Kepler would be less than $10^{-3} M_{\text {solar }}$. In this case, we no longer have a significant source of dust in the early Universe, although if the dust in the high- $z$ galaxies is also composed of iron needles, the galactic dust mass would also decrease.

Using this model, we found that the parameters required to fit the SED and observed properties of Kepler are inconsistent with those suggested for Cas A. An additional, more serious, problem with the iron needle model is that it is based on the Rayleigh criterion ( $\mathrm{Li} 2003$ ), which needs to be satisfied to produce the absorption efficiencies seen in Fig. 7. Using the range of axial ratios $(l / a<700)$ and conductivities $\left(\rho_{r} \sim(4-60) \times 10^{-17} \mathrm{~s}\right)$ required to fit Kepler's SED, the Rayleigh criterion is only satisfied for iron needles with grain radii of $0.8-5.7 \AA$. This is equivalent to approximately a few layers of iron atoms at most. It is extremely difficult to explain how such small grains with length 1000 times greater than their radius would form and indeed survive in the hot X-ray plasma.

\section{Foreground interstellar clouds}

Dunne et al. (2003b) assumed that the SCUBA dust was associated with Cas A for a number of reasons. The strongest evidence for this assumption is that the $850 \mu \mathrm{m}$ emission is completely bounded by the forward and reverse shocks of the remnant (as determined by the X-ray and radio observations). Second, they compared the sub-mm emission with the available $\mathrm{CO}$ maps of the remnant in the literature (e.g. Wilson et al. 1993; Liszt \& Lucas 1999) and found very little evidence for a correlation between the SCUBA peaks and the $\mathrm{CO}$ maps. These $\mathrm{CO}$ observations indicated highly diffuse emission over the entire remnant with a stronger concentration in the south. The sub-mm emission is not diffusely distributed outside the remnant in the same manner as the $\mathrm{CO}$ and is clumpy on small scales. Finally, they estimated the dust mass in the $\mathrm{CO}$ peaks using a gas-to-dust ratio of 150:1 and found dust masses much lower than the sub-mm emission predicted. We subsequently obtained our own $\mathrm{CO}$ maps of Cas A with the A3 receiver on the JCMT in 2004, as part of the JCMT Service Programme (see Fig. 8). The CO emission has been integrated over the velocity interval $-50 \mathrm{~km} \mathrm{~s}^{-1}<v$ $<-35 \mathrm{~km} \mathrm{~s}^{-1}$, which includes all the gas from the Perseus spiral arm. Overlaid are SCUBA contours at (a) $850 \mu \mathrm{m}$ with 


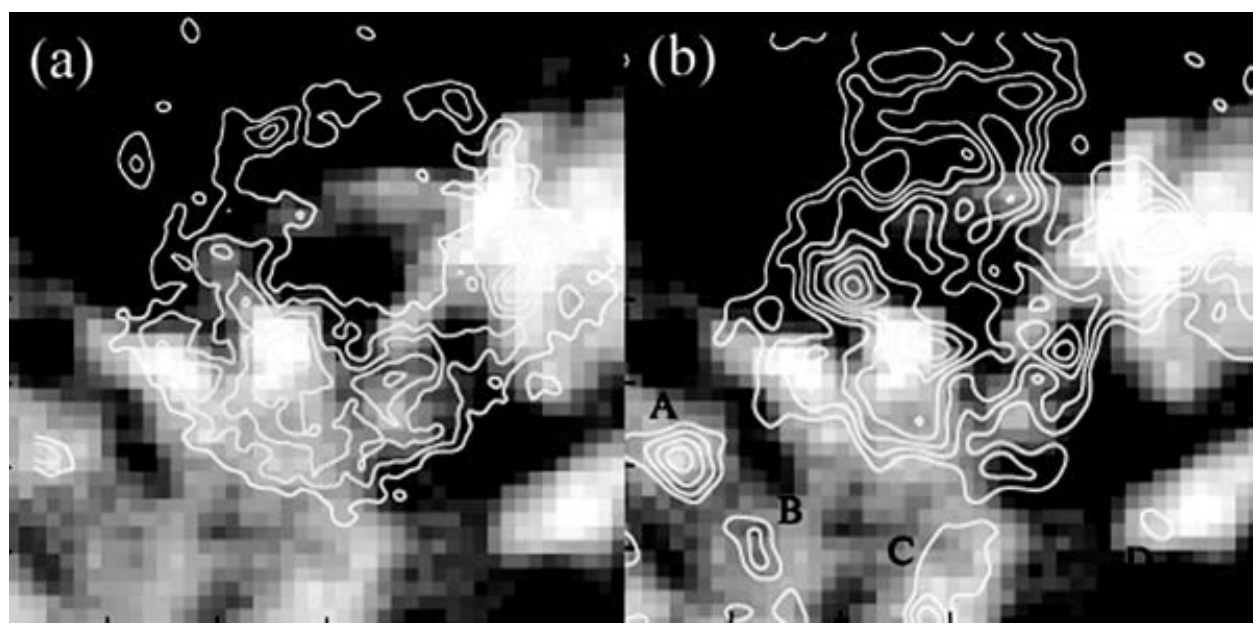

Fig. 8. Integrated $\mathrm{CO}$ emission towards Cas A over the velocity interval $-50 \mathrm{~km} \mathrm{~s}^{-1}<v<-35 \mathrm{~km} \mathrm{~s}^{-1}$, which includes all the gas from the Perseus spiral arm. Overlaid are the SCUBA contours: (a) $850 \mu \mathrm{m}$ with synchrotron emission subtracted, and (b) $450 \mu \mathrm{m}$.

synchrotron emission subtracted and (b) $450 \mu \mathrm{m}$. Some of the peaks in the sub-mm continuum are at the positions of peaks in the molecular gas, which suggests that some of the dust may be foreground material or dust that has been swept up by the blast wave. Notice also the correlation between $\mathrm{CO}$ and SCUBA dust clumps well outside the remnant (clumps A-D). These clumps are distributed in a ring-like structure centred on the remnant with radius of $290 \operatorname{arcsec}(4.8 \mathrm{pc})$ and individual sizes of $0.5-1 \mathrm{pc}$ across. The origin of these clumps is not yet understood but could have been formed in the progenitor's SW or high-velocity ejecta clumps from the supernova.

Wilson \& Bartla (2005) used CO observations towards Cas A to show positional agreement for three of the sub-mm peaks with $\mathrm{CO}$ clouds and estimated that half of the dust in the remnant could be associated with the intervening interstellar clouds. This leaves around $1 M_{\text {solar }}$ of dust in Cas A (10000 times more than detected with previous far-infrared (FIR) observations). However, uncertainties in estimating the $\mathrm{CO}$ cloud masses are large since this requires knowledge of cloud velocities and conversion factor between $\mathrm{CO}$ and molecular hydrogen (which also hinders our $\mathrm{CO}$ observations towards Kepler, see below) and large-scale observations in the sub-mm range are sorely needed to differentiate between the remnant and foreground material.

Krause et al. (2004) re-reduced the SCUBA sub-mm data and used Spitzer to observe Cas A at $160 \mu \mathrm{m}$ (Hines et al. 2004), with OH absorption emission. They used the 'median' option in removing the baseline for the $850 \mu \mathrm{m}$ data. Their final image has large negative features, which are of a greater level than the positive emission in the south. The SNR in this case is sitting on a negative background in the north and a positive background in the south. The measurement of the level of flux in the south is critical to their argument that this emission is from foreground material. They found a high degree of correlation with the dust emission and $\mathrm{OH}$ absorption seen towards the remnant (which incidentally is only detectable in absorption in the region strong in radio emission, i.e.

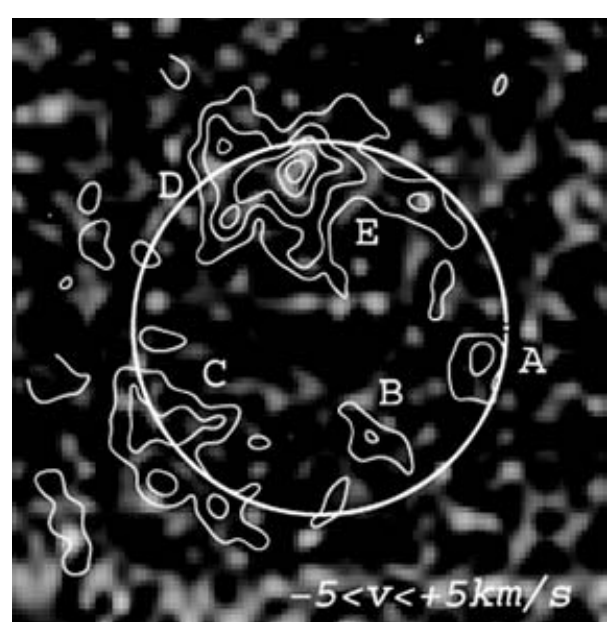

Fig. 9. Integrated carbon monoxide emission towards Kepler's supernova remnant over the velocity interval $-5<v<+5 \mathrm{~km} \mathrm{~s}^{-1}$ The $850 \mu \mathrm{m}$ SCUBA contours are shown $(3 \sigma+2 \sigma)$ along with the position of the forward shock as traced by X-ray observations. No signal is detected in these images with a $3 \sigma$ upper limit $\mathrm{I}(\mathrm{CO})$ $\sim 2.21 \mathrm{~K} \mathrm{~km} \mathrm{~s}^{-1}$. Cold dust clumps are labelled A-E.

the bounded shocks). The correlation is less convincing when compared with the $\mathrm{CO}$ emission since this has a much larger, diffuse structure. They conclude that all of the sub-mm emission is from foreground clouds. Their work suggests that no more than $0.2 M_{\text {solar }}$ of dust can be associated with the ejecta itself, although this is almost a factor of three higher if the 'normal' dust absorption coefficient value for the diffuse ISM is used when estimating the dust mass in the molecular cloud, i.e. $\sim 0.07 \mathrm{~m}^{2} \mathrm{~kg}^{-1}$ (Krause et al. (2004) use a value of $0.18 \mathrm{~m}^{2} \mathrm{~kg}^{-1}$ ). Thus, the amount of dust from the supernova Cas A or its progenitor star is very uncertain. The correlation between sub-mm continuum and molecular emission suggests that some of the dust may indeed be foreground material that has been swept up by the blast wave, but the lack of a perfect correlation between the two suggests that some of the dust is 
made in the supernova/massive star wind. We note that a substantial correction for foreground material would bring the amount of dust in Cas A better in line with the mass of dust estimated for Kepler's SNR.

Given the controversy about the amount of dust in the Cas A SNR, it is important to determine whether or not Kepler's remnant is also contaminated by foreground material. Kepler's SNR was observed in the CO $(J=2-1)$ line with the A3 receiver on the JCMT in 2004, as part of the JCMT Service Programme. We used the wide-band mode of the DAS spectrometer, which has a bandwidth of $1.8 \mathrm{GHz}$ and spectral resolution of $1.97 \mathrm{~km} \mathrm{~s}^{-1}$. We mapped a square region of $6 \times 6 \mathrm{arcmin}^{2}$. Details about the reduction process will be given in Gomez et al. (in preparation). We made no significant detection of $\mathrm{CO}$ over the entire range $-150 \mathrm{~km} \mathrm{~s}^{-1}<v<150 \mathrm{~km} \mathrm{~s}^{-1}$. Given that there is no detected signal, we can only calculate a $3 \sigma$ upper limit from the maps over the relevant velocity range of possible $\mathrm{CO}$ clouds.

Kepler's SNR is far from the Galactic plane, suggesting that confusion from foreground clouds should be easily seen and features associated with the remnant should also be easily recognizable (see, e.g., a recent $\mathrm{HI}$ study towards the remnant; Reynoso \& Goss (1999)). At lower Galactic latitudes, there is evidence for molecular cloud structures with a wide velocity range of $-20 \mathrm{~km} \mathrm{~s}^{-1}<v<+40 \mathrm{~km} \mathrm{~s}^{-1}$ although $90 \%$ of the $\mathrm{CO}$ emission in this region is within the velocity range $-10 \mathrm{~km} \mathrm{~s}^{-1}<v<+20 \mathrm{~km} \mathrm{~s}^{-1}$ (Dame et al. 2001). However, a CO latitude-velocity map at Kepler's location, shows that the velocity range of clouds at the higher latitude of Kepler's remnant is roughly $-5 \mathrm{~km} \mathrm{~s}^{-1}<$ $v<+10 \mathrm{~km} \mathrm{~s}^{-1}$ (Dame, Private communication) with most of the emission confined to the smaller range $0 \mathrm{~km} \mathrm{~s}^{-1}<v<$ $+5 \mathrm{~km} \mathrm{~s}^{-1}$ (see also Dame et al. (2001), Fig. 5(a)). Figure 9 shows the CO emission towards Kepler's SNR over the velocity range $-5 \mathrm{~km} \mathrm{~s}^{-1}<v<+5 \mathrm{~km} \mathrm{~s}^{-1}$ with the $850 \mu \mathrm{m}$ contours and the location of the shock front overlaid. Dust clumps are labelled $\mathrm{A}-\mathrm{E}$. We can determine the gas mass in the $\mathrm{CO}$ data using our upper limit $I(\mathrm{CO})$,

$M_{\mathrm{H}_{2}}=4.49 \pi R^{2} I(\mathrm{CO})$,

$M_{\text {gas }}=1.36 M_{\mathrm{H}_{2}}$

Integrating the $\mathrm{CO}$ intensity over a wide velocity range of $\Delta v \sim 30 \mathrm{~km} \mathrm{~s}^{-1}$, we estimate that the $3 \sigma$ upper limits on the dust-to-gas ratios in the clouds A-E are 48, 44, 20, 58 and 34, respectively. This is lower than the nominal 100-200 values seen in the ISM suggesting that the dust is not from a foreground molecular cloud. However, these values depend on the velocity width of clouds which could conservatively increase up to $\Delta v \sim 60 \mathrm{~km} \mathrm{~s}^{-1}$. In this case, the dust-to-gas ratios would increase by a factor of $\sqrt{60 / 30}$. Ironically, not detecting a clear signal from CO gas means we have to integrate over all possible clouds at this location to be conservative. The likelihood of a $60 \mathrm{~km} \mathrm{~s}^{-1}$ cloud is remote unless it is physically interacting with the remnant. The typical size of molecular clouds with $\Delta v \sim 60 \mathrm{~km} \mathrm{~s}^{-1}$ is greater than $30 \mathrm{pc}$
(Solomon et al. 1987), ten times larger than SCUBA clumps. Given the errors involved in the velocity width in addition to the errors in estimating the dust mass of the clouds, we require deeper observations of $\mathrm{CO}$ emission towards Kepler to obtain far better sensitivity.

\section{Conclusion}

The question of exactly how much dust is formed in $\mathrm{SNe}$ is still controversial. Chemical evolution models suggest the need for a supernova (or rapidly evolving) source of dust in both the early Universe and in our own Galaxy. In 2003, the first observational evidence of copious amounts of dust in supernova remnants was provided by SCUBA, probing the emission from cold dust, dust that previous far-infrared telescopes had missed. Other explanations for the sub-mm emission were put forward; namely (1) the emission seen in SCUBA was from 'exotic' iron needles which are efficient at radiating in the sub-mm, and (2) that the emission was actually from interstellar clouds and not the SNe themselves. Recent work suggests that one of the remnants, Cas A, is contaminated by emission from dust in a foreground cloud and, although our observations of $\mathrm{CO}$ emission from gas towards Cas A confirm that some of the dust emission may be from foreground material, this does not explain why the dust peaks in our SCUBA image fall between the bowshock and the reverse shock. This of course could be a chance alignment, but the coincidence is evidence that some of the dust was either formed in the supernova or swept up from the surrounding ISM. Our limited observations of CO emission towards Kepler so far suggest that the SCUBA emission is from dust in the remnant and not foreground material. There are many uncertainties when estimating gas masses from $\mathrm{CO}$ data, which need to be considered before this result can be verified/disproved. We conclude that even if most of the dust in Cas A and Kepler is foreground material, we may still be left with $0.1 M_{\text {solar }}$ of dust formed by the pre-supernova massive star or in the supernova blast wave. This is more than enough to explain dusty galaxies at high redshifts and solves the dust budget crisis in our own Galaxy. We eagerly await results from SCUBA-2 and the Herschel Space Observatory which will finally have the combined resolution, mapping speeds and sensitivity to resolve the question of the origin of dust in the Universe.

\section{References}

Bertoldi, F., Carilli, C., Cox, P., Fan, X., Strauss, M.A., Beelen, A., Omont, A. \& Zylka, R. (2003). Astron. Astrophys. 406, L55-L58.

Blair, W.P. (2004). Supernovae as cosmological lighthouses. In Proc. Conf. Asronomical Society of the Pacific (ASP Conference Series, vol. 342), astro-ph/0410081. ASP, San Francisco.

Borkowski, K.J., Blondin, J.M. \& Sarazin, C.L. (1992). Astrophys. J. 400, 222-237.

Braun, R. (1987). Astron. Astrophys. 171, 233-251.

Clayton, D.D., Deneault, E.A.-N. \& Meyer, B.S. (2001). Astrophys. J. 562, 480-493.

Dame, T. Private communication. 
Dame, T., Hartmann, D. \& Thaddeus, P. (2001). Astrophys. J. 547, 792-813.

Dole, H., Lagache, G., Puget, J.-L., Caputi, K.I., Fernández-Conde, N., Le Floc'h, E., Papovich, C., Pérez-González, P.G., Rieke, G.H. \& Blaylock, M. (2006). Astron. Astrophys. 451, 417-429.

Draine, B.T. (2003). The Cold Universe, pp. 73, Springer-Verlag, also available at astro-ph/0304488.

Dunne, L., Eales, S. \& Edmunds, M.G. (2003a). Mon. Not. Roy. Astron. Soc. 424, 589-598.

Dunne, L., Eales, S., Ivison, R., Morgan, H.L. \& Edmunds, M.G. (2003b), Nature 424, 285-287.

Dwek, E. (2004). Astrophys. J. 607, 848-854.

Eales, S., Bertoldi, F., Ivison, R., Carilli, C.L., Dunne, L. \& Owen, F. (2003). Mon. Not. Roy. Astron. Soc. 344, 169-180.

Edmunds, M.G. \& Wickramasinghe, C. (1975). Nature 256, 713-714.

Gomez, H.L. (née Morgan), Dunne, L., Eales, S.A., Gomez, E.L. \& Edmunds, M.G. (2005). Mon. Not. Roy. Astron. Soc. 361, 1012-1014.

Gomez et al. (in preparation).

Gotthelf, E.V., Koralesky, B., Rudnick, L., Jones, T.W., Hwang, U. \& Petre, R. (2001). Astrophys. J. 552, L39-L43.

Hildebrand, R.H. (1983). Quart. J. Roy. Astron. Soc. 24, 267-282.

Hines, D. et al. (2004). Astrophys. J. Suppl. 154, 290-295.

Hirashita, H. \& Ferrara, A. (2002). Mon. Not. Roy. Astron. Soc. 337, 921-937.

Isaak, K.G., Priddey, R.S., McMahon, R.G., Omont, A., Peroux, C., Sharp, R.G. \& Withington, S. (2002). Mon. Not. Roy. Astron. Soc. 329, 149-162.
Jones, A.P., Tielens, A.G.G.M. \& Hollenback, D.J. (1996). Astrophys. J. 469, 740-764

Kinugasa, K. \& Tsunemi, H. (1999). Pub. of the Astronomical Society of Japan 51, 239-252.

Krause, O., Birkmann, S.M., Reike, G., Lemke, D., Klaas, U., Hines, D.C \& Gordon, K.D. (2004). Nature 432, 596-598.

Li, A. (2003). Astrophys. J. 599, 380-386.

Liszt, H. \& Lucas, R. (1999). Astron. Astrophys. 347, 258-265.

Morgan, H.L., Dunne, L., Eales, S., Ivison, R. \& Edmunds, M.G. (2003). Astrophys. J. 597, L33-L36.

Morgan, H.L. \& Edmunds, M.G. (2003). Mon. Not. Roy. Astron. Soc. 343 427-442.

Nozawa, T., Kozasa, T., Umeda, H., Maeda, K. \& Nomoto, K. (2003) Astrophys. J. 598, 785-803.

Reynoso, E.M. \& Goss, W.M. (1999). Astron. J. 118, 926-929.

Schaefer, B.E. (1996). Astrophys. J. 459, 438-454.

Smail, I., Ivison, R.J. \& Blain, A.W. (1997). Astrophys. J. 490, L5-L8.

Solomon, P.M., Rivolo, A.R., Barrett, J. \& Yahil, A. (1987). Astrophys. J. 319, 730-741.

Todini, P. \& Ferrara, A. (2001). Mon. Not. Roy. Astron. Soc. 325, 726-736.

Tuffs, R., Fischera, J., Drury, L.O'C., Gabriel, C., Heinrichsen, I., Rasmussed, I. \& Volk, H.J. (1999). The Universe as Seen by ISO, vol. 427, ed Cox, P. \& Kessler, M.F., pp. 241-245.

Whittet, D.C.B. (2003). Dust in the Galactic Environment, 2nd edn, pp. 258-259. IOP, Bristol.

Wilson, T.L. \& Batrla, W. (2005). Astron. Astrophys. 430, 561-566.

Wilson, T.L., Mauersberger, R., Muders, D., Przewodnik, A. \& Olano, C.A. (1993). Astron. Astrophys. 280, 221-230. 